ECCOMAS

\section{Proceedia}

COMPDYN 2017

$6^{\text {th }}$ ECCOMAS Thematic Conference on Computational Methods in Structural Dynamics and Earthquake Engineering

M. Papadrakakis, M. Fragiadakis (eds.) Rhodes Island, Greece, 15-17 June 2017

\title{
SEISMIC ASSESSMENT OF AN UNCONVENTIONAL STRUCTURE: THE CRYOSTAT OF “CUORE" EXPERIMENT
}

\author{
Raffaele Ardito $^{1}$, Oliviero Cremonesi ${ }^{2}$, and Federico Perotti ${ }^{1}$ \\ ${ }^{1}$ Department of Civil and Environmental Engineering, Politecnico di Milano \\ Piazza Leonardo da Vinci 32, 20154 Milan, Italy \\ \{raffaele.ardito, federico.perotti\}@polimi.it \\ ${ }^{2}$ Istituto Nazionale di Fisica Nucleare INFN - Sez. di Milano-Bicocca \\ Piazza della Scienza 3, 20126 Milan, Italy \\ oliviero.cremonesi@mib.infn.it
}

Keywords: Structural Dynamics, Earthquake Engineering, Contact Mechanics, Explicit Algorithms, Seismic Isolation.

\begin{abstract}
This paper deals with the seismic assessment of a complex structure supporting the CUORE experiment (Cryogenic Underground Observatory for Rare Events). The main issues, that require a special computational treatment, are: (i) non-linear geometric behavior of the suspended vessels; (ii) multiple contacts between the nested vessels and between the cryostat and the external shields; (iii) non-linear constitutive law of the HDR bearings. The problem has been tackled by building a 3D detailed finite element model, that has been used for simulating, via an explicit step-by-step algorithm, the dynamic response to artificial spectrumcompatible accelerograms. The results of the analyses have been used for introducing some specific structural improvements.
\end{abstract}




\section{INTRODUCTION}

The CUORE experiment (Cryogenic Underground Observatory for Rare Events, see [1, 2]) is a nuclear physics experiment funded by several agencies, among which the National Italian Institute of Nuclear Physics (INFN), the US Department of Energy (DOE) and the National Science Foundation (NSF, USA). The experiment is set up in the Gran Sasso National (Italian) Laboratory (LNGS), an underground facility in a highly seismic zone. In fact, the laboratory is located in a gallery beneath the Gran Sasso mountain, not far from the city of L'Aquila: that zone has been hit by a destructive earthquake in 2009 (MMS 6.3) and is currently involved in the earthquake swarm that follows the Amatrice earthquake (August 2016, MMS 6.2). In spite of the fact that the underground gallery seems to mitigate the seismic effects $[3,4]$, there is a major concern on the structural safety of all the experiments hosted by LNGS. The complexity of the structures, along with the strict requirements from the point of view of experimental physics, calls for the application of computational tools at the cutting-edge of technology.

The main purpose of the CUORE experiment is the search for neutrinoless double beta decay, with many important consequences on particle physics and astrophysics. The heart of the experiment is a detector (i.e. a large mass of $\mathrm{TeO}_{2}$ crystals) that is contained in a cryostat of exceptional performance [5,6]: indeed, the detector is cooled down until absolute temperatures lower than $10 \mathrm{mK}$. A base temperature lower than $8 \mathrm{mK}$ has actually been reached on January 30, 2017. The thermal shield system is represented by a set of nested vessels, whose structural behavior resembles to that of pendulums. Moreover, the protection from physical interferences (cosmic rays and environmental radioactivity) requires the presence of a set of lead shields and of a borated polyethylene cap. The cryostat hangs from a steel frame, that is based on a r.c. structure supported by a base isolation system. The r.c. structure, that is clearly visible in Figure 1, is composed of two parallel walls, connected by three horizontal beams. The walls are supported by four elastomeric bearings (High Damping Rubber Bearings, HDRB).

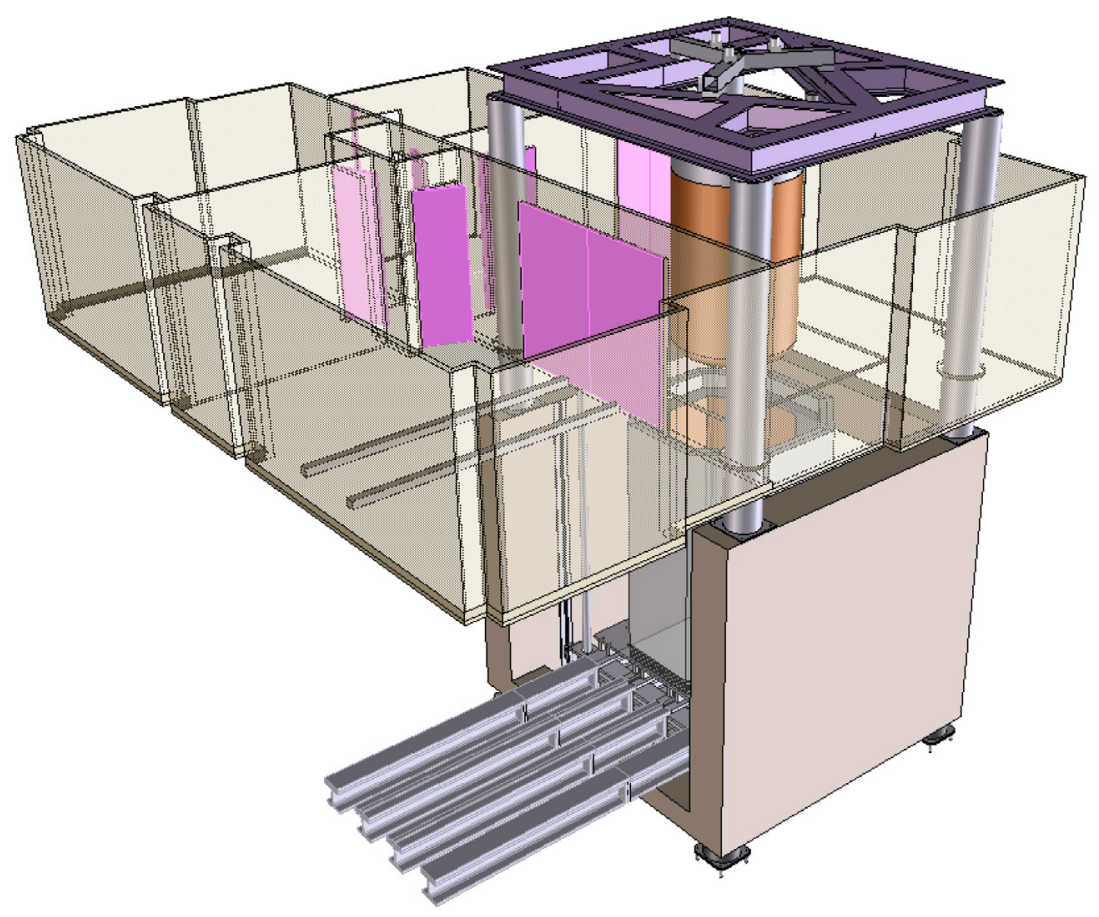

Figure 1: Rendering of the structure that contains the CUORE experiment. The external vessel of the cryostat is visible since the lead shield is completely lowered. 

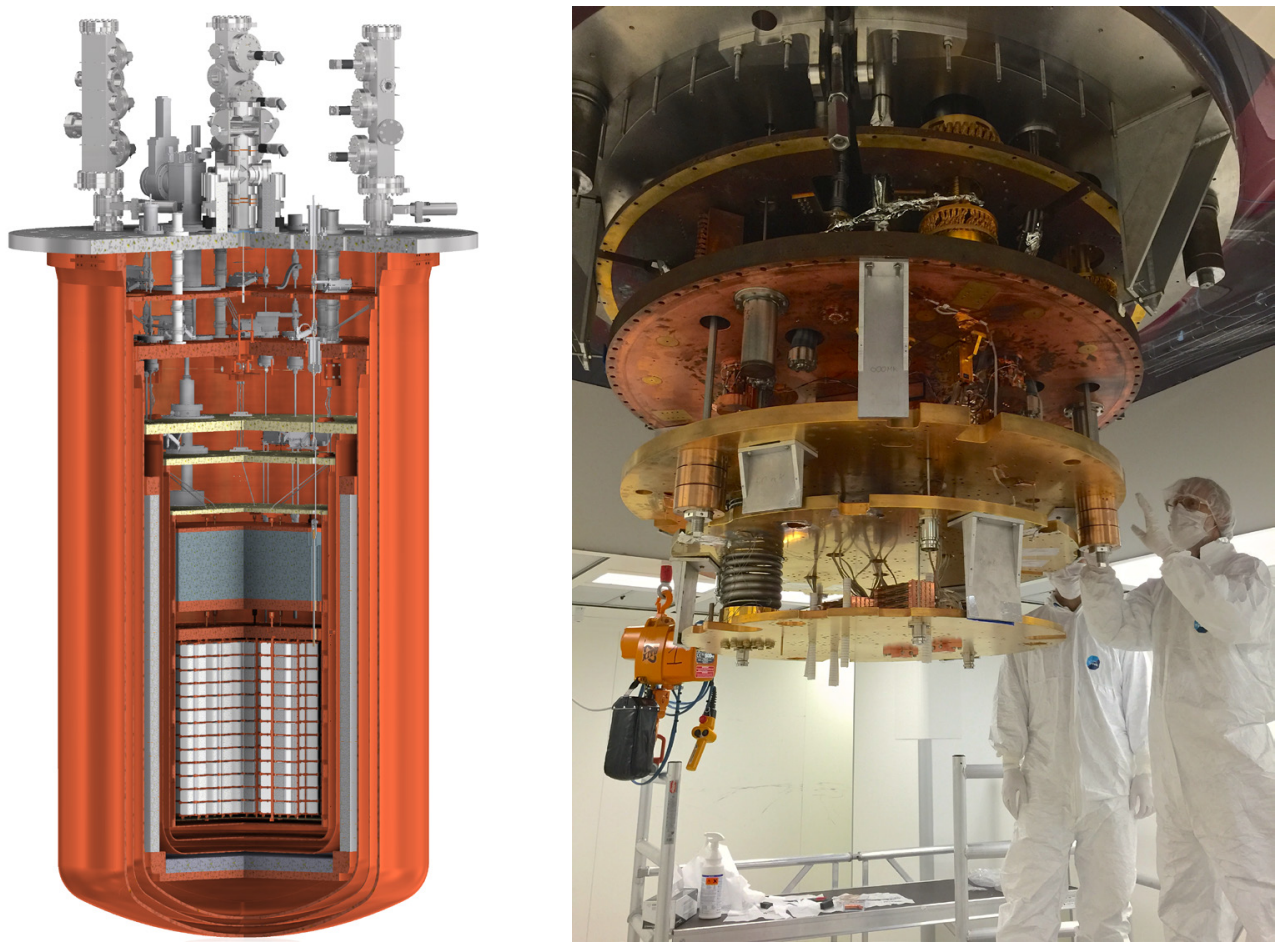

Figure 2: On the left, cut-out of the CUORE cryostat, with the inner detector constituted of about $1000 \mathrm{TeO}_{2}$ crystals. On the right, actual view of some of the cryostat's flanges.

In the operative configuration, the external lead shield is raised so that it covers completely the cryostat. The shield, that is placed on a very stiff steel plate raised and lowered by four threaded bars (jacks), is connected to the four steel columns by means of eight steel ties. The cryostat, that is depicted in Figure 2, is attached, by means of steel ropes, to the horizontal framed structure that is visible on the top of the columns in Figure 1. The flanges of each vessel are connected to one another by means of copper rods. The gap between two consecutive vessels is around one centimeter so that the mutual contact is likely to occur during seismic excitation. The total mass of the cryostat (with the detector) is about $18 \mathrm{t}$. The mass of the external lead shield is $75 \mathrm{t}$, and the mass of the steel and r.c. members is $85 \mathrm{t}$.

\section{COMPUTATIONAL MODEL}

The analyses have been carried out by means of the Finite Element method, building a 3D detailed model, that has been used for carrying out step-by-step dynamic analyses of the response to artificial accelerograms. A schematic view of the model is depicted in Figure 3. The concrete walls have been modeled by means of shell elements (constant strain triangles), whereas the steel columns and the other steel members have been discretized by Timoshenko beam elements. In order to ease the computational burden, the various vessels of the cryostat, the detector and the external shields have been modeled as rigid parts. Conversely, the suspensions of the cryostat's elements have been introduced as Timoshenko beams or truss elements, according to the specific constraints. Finally, the HDR bearings have been represented by means of non-linear springs with different behavior in the vertical and the horizontal directions.

The mechanical response is intrinsically non-linear for several reasons: (i) the rubber of the isolator is a non-linear elastic material; (ii) the cryostat is basically constituted of several nested pendulums, whose dynamic behavior is properly caught only by analyses with non-linear geometry; (iii) ideally hard and frictionless contacts should be envisaged between the vessels. 


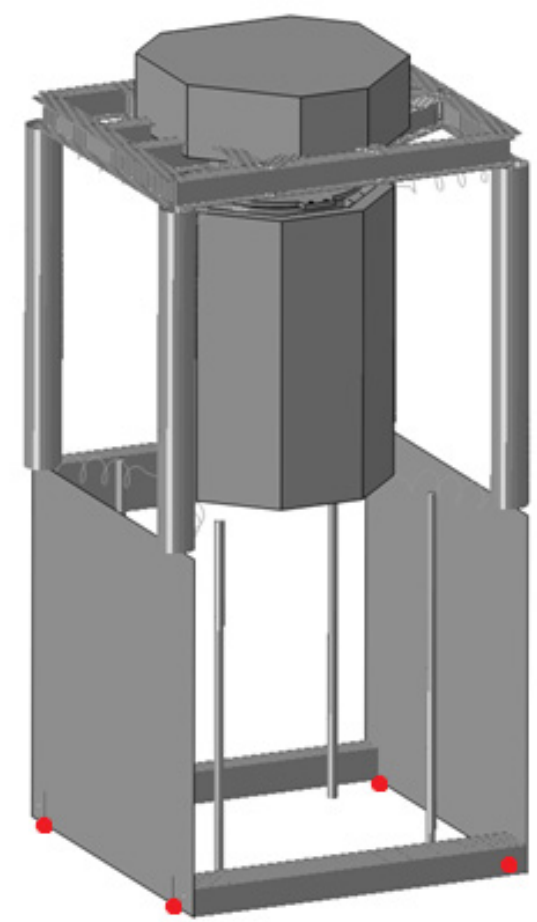

Figure 3: Schematic view of the model that has been adopted for the analyses.

For the above reasons, the explicit scheme of integration has been chosen. Such an algorithm, though conditionally stable [7], is perfectly suited to handle the occurrence of severe internal and external contacts. The stable time increment has been evaluated on the basis of the wave propagation modes in the smallest finite element; in the present case, the mass scaling procedure does not entail significant benefits.

The mechanical behavior of the elastomeric bearings has been simulated by means of the Fujino model $[8,9]$. In this way, it was possible to capture the hysteretic behavior of rubber, represented in Figure 4. It is worth noting that the preliminary analyses have been carried out by considering a linearized version of the constitutive model: the secant stiffness has been obtained by means of an iterative procedure, that usually converges in no more than 6 iterations.

\section{MAIN RESULTS OF THE ANALYSES}

The step-by-step analyses have been preceded by a set of spectrum response analysis, with the purpose of obtaining some general information on the mechanical behavior. The eigenvalue extraction included the linearized geometric effects, so that the correct "small oscillation" frequency of each pendulums has been achieved. The base isolators have been linearized by means of the abovementioned iterative procedure. The contact between vessels cannot be reproduced by means of linear analyses. An approximate solution can be obtained if one assumes that, in view of the close proximity of the nested vessels, the cryostat behaves as a unique rigid body. Such a procedure has yielded acceptable results for seismic intensity corresponding to the ultimate limit state. The first natural frequencies for the isolated structure are equal to $1.1138 \mathrm{~Hz}$ and $1.2305 \mathrm{~Hz}$, in the direction orthogonal and parallel to the concrete walls, respectively. The earthquake response spectrum has been selected according to the Italian seismic code: the peak ground acceleration is $0.261 \mathrm{~g}$ and the maximum spectral acceleration is $0.616 \mathrm{~g}$, in the case of $5 \%$ damping. The natural frequencies fall in the constant velocity branch of the response spectrum, as it is desirable for an isolated structure. The max- 
imum displacement of the isolators is about $38 \mathrm{~mm}$, which means that the working point corresponds to an overall angular strain about 30\%. The strain level is rather low: indeed, the original structural design, which was executed by an independent professional engineer, introduced largely oversized isolators.

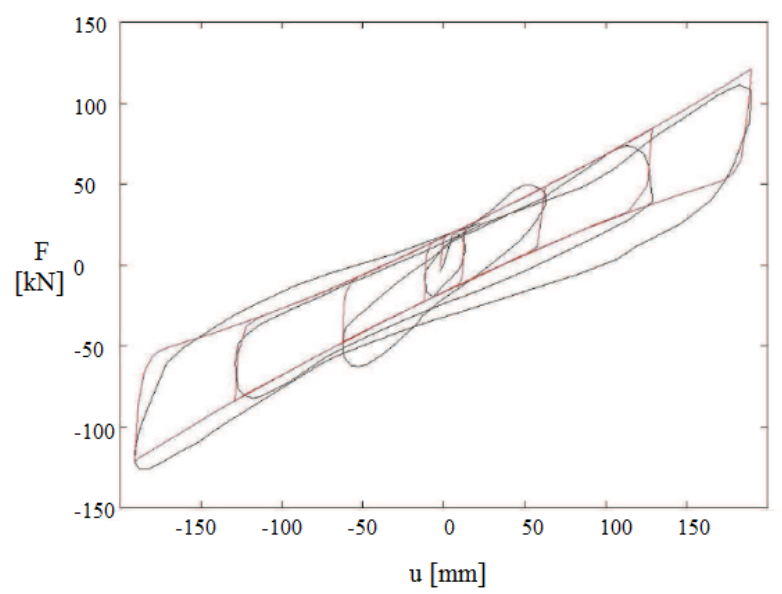

Figure 4: Hysteretic behavior of the HDRB: experimental results (black) vs. Fujino model (red).

As an example of the results achieved by the response spectrum analyses, Figure 5 shows the contour plot of horizontal displacements in the support structure. The maximum displacement is about $55 \mathrm{~mm}$.

The time-marching analyses have been based on artificial accelerograms, generated on the basis of the same response spectrum that has been used for the linearized model. An example of the artificial accelerogram is reported in Figure 6. The analyses included the non-linear behavior both for geometric effects and for material behavior of the isolator. Moreover, hard and frictionless contact has been established between the cryostat's vessels and between the cryostat and the external lead shield. The complexity of the analysis is very high, as well as the computational burden.

A typical feature of the non-linear step-by-step analyses was the occurrence of extremely high acceleration peaks: that was due to the occurrence of pounding between rigid parts. On the other hand, the displacement pattern was in satisfactory agreement with the preliminary linearized analyses. Figure 7 shows that the maximum horizontal displacements is about 35 $\mathrm{mm}$. The displacement for linearized analyses was slightly larger, but this is an expected outcome for systems having intermediate-to-low natural frequencies. Moreover, the linearized analyses were based on the hypothesis of rigidity of the whole cryostat, that is clearly surpassed by the non-linear analyses. The effect of internal contacts is clearly visible if one examines the absolute displacement and the acceleration of the vessels: the impacts correspond to acceleration peaks and to abrupt change of the displacement pattern.

\section{CONCLUSIONS}

The paper has presented the computational details for the seismic analysis of a complex structure, used for an experiment in nuclear physics. The strongly non-linear behavior has been dealt with through explicit step-by-step analyses, whose results have been critically compared to the outcomes of a simplified linearized model. 

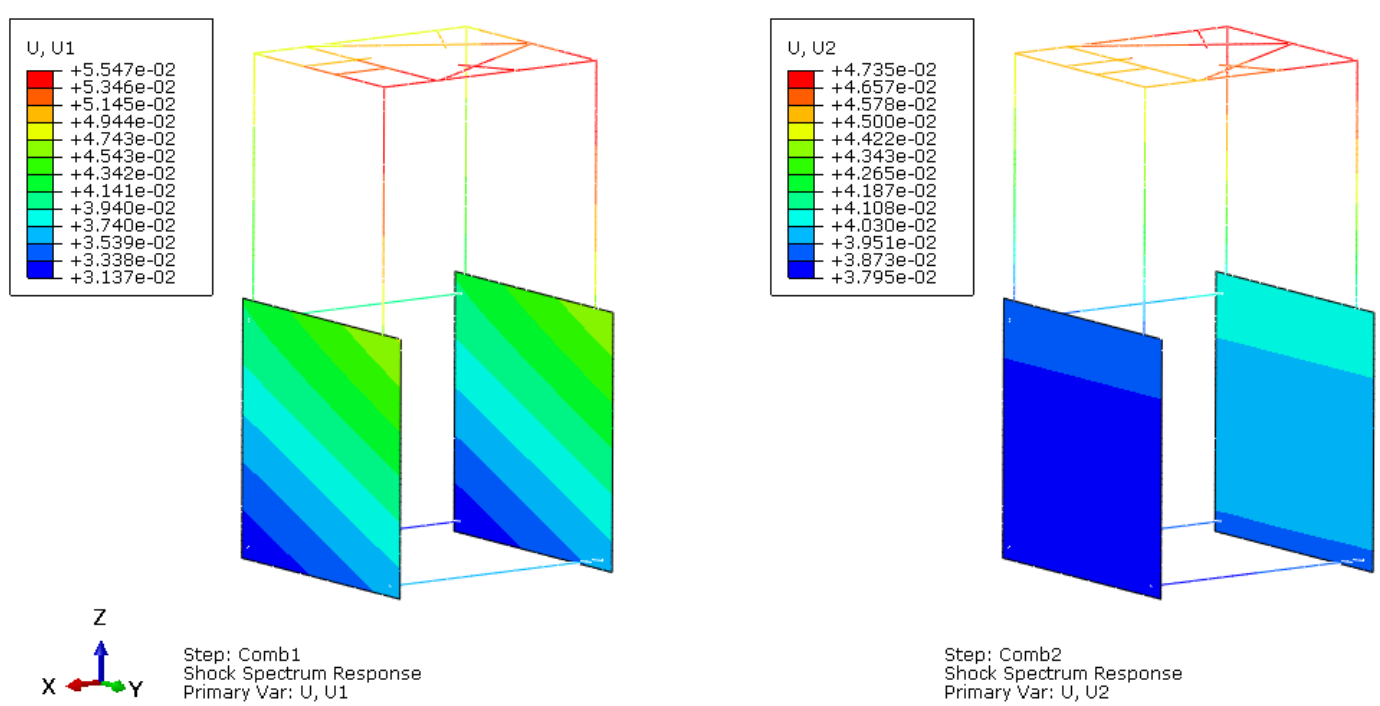

Step: Comb2 Shock Spectrum Response

Figure 5: Results of the response spectrum analyses: horizontal displacement in the orthogonal (left) and parallel (right) direction with respect to the concrete walls.

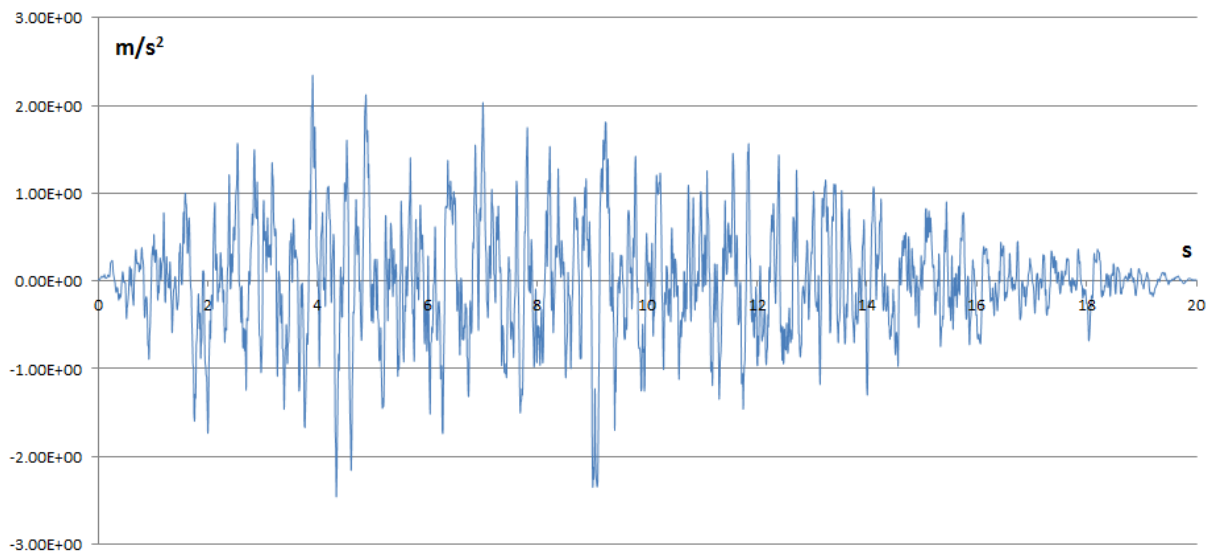

Figure 6: Typical accelerogram used for the time-marching analysis. The accelerogram has been artificially generated on the basis of the elastic response spectrum provided by the Italian seismic regulation.

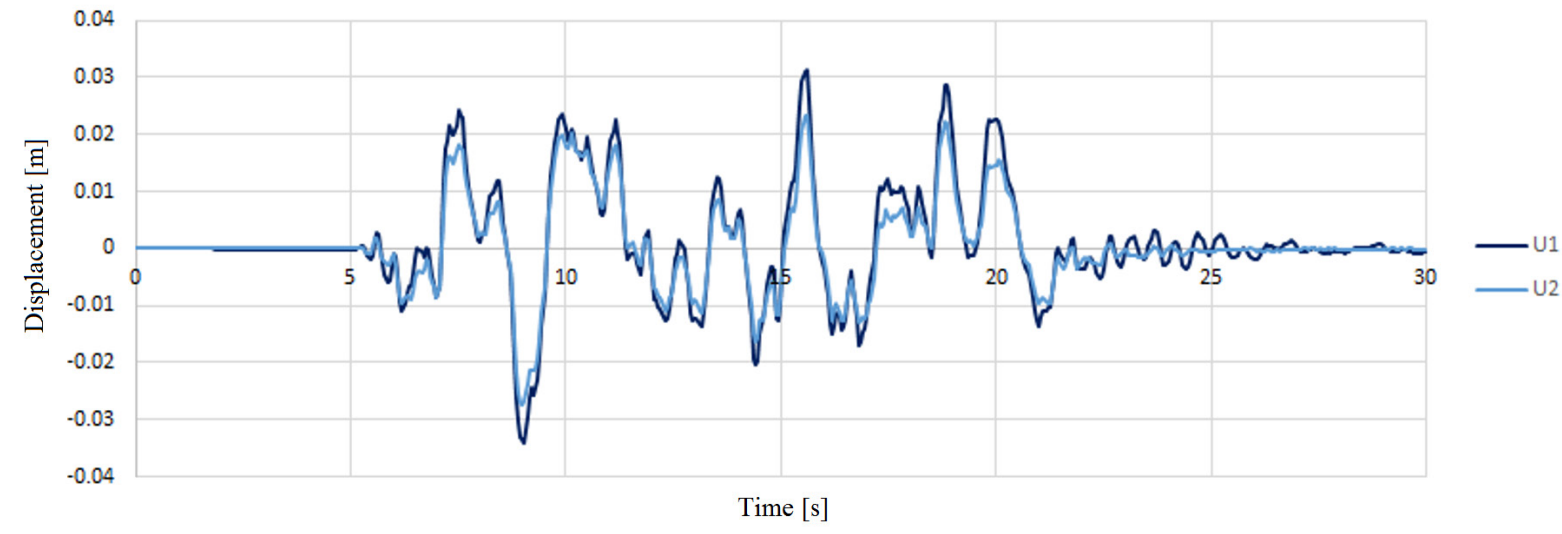

Figure 7: Time history of the horizontal displacement for the non-linear analyses. 
The complete set of results, which have not been reported herein for the sake of brevity, have been thoroughly examined in order to assess the seismic safety of the structure. It is worth noting that, on one hand, the structural layout should guarantee the typical decoupling of isolated structures under seismic actions. On the other hand, in service conditions, a full connection to ground should ensure the correct irradiation of internal vibrational energy due to the pumping systems and to the cryocoolers. For the ultimate limit state, the analyses have shown a critical behavior in correspondence of the r.c. beam which connects the two concrete walls. The as-built reinforcement does not seem to match the ductility and strength requirements, so that a specific seismic retrofitting is required. After several attempts, the best solution seems to be represented by the introduction of a K-shaped or X-shaped bracing system, that provides the suitable resistant mechanism in the orthogonal direction with respect to the concrete walls. The linear and non-linear analyses confirm that such a provision is able to establish the correct seismic behavior.

\section{REFERENCES}

[1] R. Ardito et al., The CUORICINO and CUORE double beta decay experiments, Progress in Particle and Nuclear Physics, 57, 203-216, 2006.

[2] F. Alessandria et al., CUORE crystal validation runs: Results on radioactive contamination and extrapolation to CUORE background, Astroparticle Physics, 35, 839-849, 2012.

[3] D. Kolymbas, Tunneling and Tunnel Mechanics, Springer, 2008.

[4] A. Castellani, G. Canetta, S. Pace, R. Guidotti The vaults of the Gran Sasso National Laboratory Halls, during the 2009 L'Aquila earthquake. Design of a monitoring system against earthquakes (in Italian), Gallerie e Grandi Opere Sotterranee, 103, 25-39, 2012.

[5] A. Nucciotti et al., Design of the cryogen-free cryogenic system for the CUORE experiment, Journal of Low Temperature Physics, 151, 662-668, 2008.

[6] F. Alessandria et al., The $4 \mathrm{~K}$ outer cryostat for the CUORE experiment: Construction and quality control, Nuclear Instruments and Methods in Physics Research Section A, 727, 65-72, 2013.

[7] K.-J. Bathe, Finite Element Procedures, Prentice-Hall, 1996.

[8] M. Abe, J. Yoshida, Y. Fujino, Multiaxial behaviors of laminated rubber bearings and their modeling. I: Experimental study, Journal of Structural Engineering, 130, 11191132, 2004.

[9] M. Abe, J. Yoshida, Y. Fujino, Multiaxial behaviors of laminated rubber bearings and their modeling. II: Modeling, Journal of Structural Engineering, 130, 1133-1144, 2004. 